\title{
Some considerations on topologies of infinite dimensional unitary coadjoint orbits.*
}

\author{
Pavel Bóna \\ e-mail: bona@sophia.dtp.fmph.uniba.sk \\ Department of Theoretical Physics, Comenius University \\ SK-842 48 Bratislava, Slovakia
}

July 17, 2021

\begin{abstract}
The topology of the embedding of the coadjoint orbits of the unitary group $\mathcal{U}(\mathcal{H})$ of an infinite dimensional complex Hilbert space $\mathcal{H}$, as canonically determined subsets of the B-space $\mathfrak{T}_{s}$ of symmetric trace class operators, is investigated. The space $\mathfrak{T}_{s}$ is identified with the B-space predual of the Lie-algebra $\mathcal{L}(\mathcal{H})_{s}$ of the Lie group $\mathcal{U}(\mathcal{H})$. It is proved, that orbits consisting of symmetric operators with finite rank are (regularly embedded) closed submanifolds of $\mathfrak{T}_{s}$. An alternative method of proving this fact is given for the "one-dimensional" orbit, i.e. for the projective Hilbert space $P(\mathcal{H})$. Also a technical assertion concerning existence of simply related decompositions into one-dimensional projections of two unitary equivalent (orthogonal) projections in 'generic relative position' is formulated, proved, and illustrated.
\end{abstract}

\section{Introduction}

Mathematical formalism of non-Einstein-relativistic quantum mechanics (QM) is traditionally based on separable complex Hilbert space $\mathcal{H}$, and on closely connected ob-

${ }^{*}$ This is a revised version of the preceding versions of this paper. The main difference consists of the correction of a wrong assertion of the Lemma 3.1 based on an erroneous assumption. The error was discovered in [12, Sec. 5]. 
jects: The $C^{*}$-algebra of bounded operators $\mathcal{L}(\mathcal{H})$, the $\sigma\left(\mathcal{L}(\mathcal{H}), \mathcal{L}(\mathcal{H})_{*}\right)$-continuous (with $\mathcal{L}(\mathcal{H})_{*}:=\mathfrak{T}:=L^{1}(\mathcal{H}):=$ the trace-class operators on $\left.\mathcal{H}\right)$ linear functionals on $\mathcal{L}(\mathcal{H})$ (identified with $\nu \in \mathfrak{T}$ ), and the group of $*$-automorphisms *-Aut $(\mathcal{L}(\mathcal{H}))$ of $\mathcal{L}(\mathcal{H})$ (acting on linear functionals by the transposed maps). Dynamics (i.e. time evolution) and symmetries of physical systems are described by subgroups of the automorphism group of $\mathcal{L}(\mathcal{H})$. Since each automorphism $\alpha \in{ }^{*}$-Aut $(\mathcal{L}(\mathcal{H}))$ of the $C^{*}$-algebra $\mathcal{L}(\mathcal{H})$ is inner, it is described by a unitary operator $(\mathcal{U}(\mathcal{H})$ is the set of all unitary elements of $\mathcal{L}(\mathcal{H}))$ $\mathrm{u}_{\alpha} \in \mathcal{U}(\mathcal{H}): \alpha(B)=\mathrm{u}_{\alpha} B \mathrm{u}_{\alpha}^{*}$ ( $\mathrm{u}_{\alpha}$ is determined by $\alpha$ up to a numerical factor). In physics, symmetries and dynamics are modelled by Lie groups $G$. In the traditional linear QM, Lie groups are represented by strongly continuous unitary (or projective) representations $g(\in G) \mapsto U(g)(\in \mathcal{U}(\mathcal{H}))$, hence linear dynamics is described by one parameter unitary groups $U_{t} \equiv \exp (-i t H)$, with a selfadjoint operator $H$ on (a dense domain of) $\mathcal{H}$. Since physically interesting objects describing "states" are not vectors $x \in \mathcal{H}$, but to the vectors $x$ corresponding one-dimensional projectors $P_{x}$ onto the subspaces of $\mathcal{H}$ containing $x$, as well as their convex combinations (so called mixed states described by 'density matrices' $\left.\rho:=\sum_{j} \lambda_{j} P_{x_{j}} \in \mathcal{S}_{*}:=\left\{\rho \in \mathfrak{T}_{s}: \rho \geq 0, \operatorname{Tr} \rho=1\right\} \subset \mathfrak{T}_{s}\right)$ the physically interesting orbits of actions of the considered groups (resp. their representations) are orbits of the coadjoint action of $\mathcal{U}(\mathcal{H})$ (and of its subgroups), considered as a Lie group (see below).

In a more general (also nonlinear) setting, cf. e.g. [3], symmetries and dynamics in such an "extended quantum mechanics" (EQM) can be described by unitary cocycles $(g ; \rho)\left(\in G \times \mathcal{S}_{*}\right) \mapsto \mathrm{u}_{Q}(g, \rho)(\in \mathcal{U}(\mathcal{H})), \mathrm{u}_{Q}\left(g_{1} \cdot g_{2}, \rho\right)=\mathrm{u}_{Q}\left(g_{1}, \phi_{g_{2}}^{\mathrm{Q}}(\rho)\right) \cdot \mathrm{u}_{Q}\left(g_{2}, \rho\right)$, acting on the set $\mathcal{S}_{*}$ of all 'density matrices', again by means of the coadjoint action of $\mathcal{U}(\mathcal{H})$, i.e. $\phi^{\mathrm{Q}}:(g ; \rho)\left(\in G \times \mathcal{S}_{*}\right) \mapsto \phi_{g}^{\mathrm{Q}}(\rho):=\mathrm{u}_{Q}(g, \rho) \cdot \rho \cdot \mathrm{u}_{Q}(g, \rho)^{-1}$ (here Q is, in the case of one-parameter group $G:=\mathbb{R}$, a Hamiltonian function given on a Poisson manifold, specifying the cocycle $\mathrm{u}_{\mathrm{Q}}$ ). These actions leave the orbits of the coadjoint representation $A d^{*}(\mathcal{U}(\mathcal{H}))$ going through $\rho \in \mathcal{S}_{*}$ again invariant, leaving invariant also the whole set $\mathcal{S}_{*}$. This EQM is a general scheme of theories including Hamilton classical mechanics (CM), linear QM, and also various versions of nonlinear QM, and also other in physics used theoretical schemes as, e.g., various nonlinear "approximations" to QM (e.g., the time dependent Hartree-Fock theory).

These remarks have to stress that the coadjoint orbits of the Lie group (see below) $\mathcal{U}(\mathcal{H})$ going through symmetric trace class operators are important mathematical objects in physical description of a rather large scale of "processes".

As I have learned from a discussion with colleagues Anatol Odzijewicz and Tudor Ratiu, there is an "innocently looking" question connected with a work with coadjoint action of Lie groups, which is far not trivial in the general case. It is the question in 
which way the homogeneous spaces $G / G_{\rho}$ of a Lie group $G$ with their natural analytic manifold structure (with $G_{\rho}$ being the stability subgroup of $G$ at the point $\rho$ ), specifically their coadjoint orbits, are included into the topological spaces where the group acts. In more specific terms the question is, whether the injective inclusion is an immersion and homeomorphism of the analytic manifold $G / G_{\rho}$ onto a submanifold of the space $\mathcal{T}$ on which the group $G$ acts. E.g., an orbit $\mathcal{O}$ of a specific action of $\mathbb{R}$ on the two-torus $T^{2}=S^{1} \times S^{1}$, i.e. $\mathcal{O}:=\left\{\left(e^{i t \omega_{1}} ; e^{i t \omega_{2}}\right): t \in \mathbb{R}\right\} \subset T^{2}$ with irrational quotient $\omega_{1} / \omega_{2}$, covers the torus densely, hence it is not a submanifold of $T^{2}$. As it is shown in a Kirillov's example [2] (cited and reproduced in [1, 14.1.(f), p.449]), such a pathologically looking case is possible also in the cases of finite-dimensional coadjoint orbits. The more one could expect such a phenomenon in the case of infinite-dimensional orbits of Banach Lie groups.

Let $\mathcal{O}_{\rho}(\mathfrak{U})=\mathfrak{U} / \mathfrak{U}_{\rho}$ be the homogeneous space of the unitary group $\mathfrak{U}:=\mathcal{U}(\mathcal{H})$ of the infinite-dimensional Hilbert space $\mathcal{H}$ corresponding to an orbit of the action $\mathrm{u} \mapsto \mathrm{u} \rho \mathrm{u}^{*}$ on the space $\mathfrak{T}_{s}(\ni \rho)$ of symmetric trace class operators in $\mathcal{L}(\mathcal{H})$. The space $\mathfrak{T}_{s}$ is naturally identified with the predual $\mathcal{L}(\mathcal{H})_{s *}$ of the Lie algebra $\operatorname{Lie} \mathcal{U}(\mathcal{H}):=i \mathcal{L}(\mathcal{H})_{s} \sim \mathcal{L}(\mathcal{H})_{s}\left(\mathfrak{U}_{\rho}\right.$ is the stability subgroup of $\mathfrak{U}$ at $\rho$, namely $\mathfrak{U}_{\rho}=\{\rho\}^{\prime} \cap \mathfrak{U}, \rho^{*}=\rho \in \mathfrak{T}_{s}$, with $\{A\}^{\prime}$ being the commutant in $\mathcal{L}(\mathcal{H})$ of $A$ ).

In the paper [3], the topology of the orbits $\mathcal{O}_{\rho}(\mathfrak{U})$, as well as the topology of their natural injection into the dual B-space (containing the predual $\mathfrak{T}_{s}$ ) were investigated. ${ }^{1}$ It was proved there (cf. [3, Proposition 2.1.5]), that orbits trough symmetric traceclass operators are injectively immersed into $\mathfrak{T}_{s}$ iff they are going trough operators with finite rank. There was not completed, however, the proof of an assertion on regularity of this embedding (in the terminology of [9]) of such "finite-rank" orbits, which claim was contained in the text of the Proposition 2.1.5. ${ }^{2}$ One of the aims of this paper is to fill this gap.

Let us note, that the posed question of whether the orbit is also a submanifold of the "ambient" space in which the group acts is easily and positively answered in the case of finite-dimensional Hilbert space $\mathcal{H}$ : In that case the group $\mathcal{U}(\mathcal{H})$ is compact, so that the orbits are also compact and a continuous bijection of any compact space into a Hausdorff space is a closed mapping, hence a homeomorphism. For an infinite-dimensional

\footnotetext{
${ }^{1}$ Let us note that a far reaching generalization of some of structures developed and investigated in [3] is contained in very elegant paper [5]; that paper was also stimulating for the here reported research.

${ }^{2}$ The claim of "regularity of the embedding" was, however, superfluous (nevertheless correct, as it could be seen from what we shall prove here) with respect to the validity of the Proposition 2.1.5 (without the requirement of regularity of the embedding in its item (iv)), as well as with respect to its actual applications in all the paper [3].
} 
$\mathcal{H}$, however, the orbits $\mathcal{O}_{\rho}(\mathfrak{U})$ are noncompact.

The proof of the main theorem is contained in the next Section 2. The presented proof is based on a simple idea, and it does not contain any "sophisticated mathematics"; it needed just some linear algebra and elementary topology to be presented in details. In the last Section 3 some additional facts (including a proof of the fact that the orbits consisting of finite-rank density matrices are closed subsets of the "ambient" space) are presented. Also an independent proof of regularity of the embedding (we use here the definitions adopted from [9] differing from those introduced in [1], also to keep continuity with [3]) of the projective Hilbert space is presented: It indicates also an alternative way for proving the main Theorem 2.6 for the general case.

\section{A proof of regularity of the embedding}

We shall accept here some conventions and results from [3], mainly from the proof of Proposition 2.1.5 and Theorem 2.1.19. The proof of the following Theorem 2.6 completes the missing part of the proof of Proposition 2.1.5 in [3] concerned the regularity of the embedding of $\mathcal{O}_{\rho}(\mathfrak{U}) \subset \mathfrak{T}_{s}$. Some of the constructions built and used in the run of the proof might be, perhaps, also of independent interest.

Let us describe first in more details a formulation of the problem, and our strategy to approach it. It is known, [6, Proposition 37, Chap. III.§3], that the unitary group $\mathfrak{U}:=$ $\mathcal{U}(\mathcal{H})$ of the $C^{*}$-algebra $\mathcal{L}(\mathcal{H})$ of all bounded operators on a complex Hilbert space $\mathcal{H}$ is a Banach Lie group, and its Lie algebra Lie $(\mathfrak{U})$ consists of all antisymmetric bounded linear operators $i \mathcal{L}(\mathcal{H})_{s}$, which is B-space isomorphic to $\mathcal{L}(\mathcal{H})_{s}$. The adjoint representation of $\mathfrak{U}$ in the B-space $\mathcal{L}(\mathcal{H})_{s}$ is $A d: \mathfrak{U} \rightarrow \mathcal{L}\left(\mathcal{L}(\mathcal{H})_{s}\right), \mathrm{u} \mapsto A d(\mathrm{u})$, with $A d(\mathrm{u}) B:=\mathrm{u} B \mathrm{u}^{*}, \forall B \in$ $\mathcal{L}(\mathcal{H})_{s}$. The representation we are mostly interested in here is the coadjoint representation consisting of the transposed mappings $A d^{*}(\mathrm{u}):=A d\left(\mathrm{u}^{-1}\right)^{*}$ to $A d\left(\mathrm{u}^{-1}\right)^{\prime} \mathrm{s}$, hence acting on continuous linear functionals $\nu \in \mathcal{L}(\mathcal{H})_{s}^{*}, \nu: \mathcal{L}(\mathcal{H})_{s} \rightarrow \mathbb{C}, B \mapsto\langle\nu ; B\rangle$; the mapping $A d^{*}(\mathrm{u}): \mathcal{L}(\mathcal{H})_{s}^{*} \rightarrow \mathcal{L}(\mathcal{H})_{s}^{*}$ is determined by $\left\langle A d^{*}(\mathrm{u}) \nu ; B\right\rangle:=\left\langle\nu ; A d\left(\mathrm{u}^{-1}\right) B\right\rangle$. The subset of symmetric normal linear functionals can be identified with the B-space $\mathfrak{T}_{s} \subset \mathcal{L}(\mathcal{H})_{s}^{*}$ of symmetric trace-class operators : $\nu\left(\in \mathfrak{T}_{s}\right): B \mapsto\langle\nu ; B\rangle:=\operatorname{Tr}(\nu B)$; the space of normal (i.e. continuous in the topology on $\mathcal{L}(\mathcal{H})$ given by the seminorms $p_{\nu}: B \mapsto$ $\left.p_{\nu}(B):=|\operatorname{Tr}(\nu B)|, \nu \in \mathfrak{T}_{s}\right)$ symmetric functionals is a Banach space $\mathfrak{T}_{s}$ with the tracenorm $\|\nu\|_{1}:=\operatorname{Tr}|\nu|$, with the absolute value of the operator $\nu$ defined as the operator $|\nu|:=\sqrt{\nu^{*} \nu} \in \mathcal{L}(\mathcal{H})$.

We are interested in comparison of two topologies introduced on the orbits $\mathcal{O}_{\rho}(\mathfrak{U}):=$ 
$\left\{\mathrm{u} \rho \mathrm{u}^{*}: \mathrm{u}^{-1}=\mathrm{u}^{*} \in \mathfrak{U} \subset \mathcal{L}(\mathcal{H})\right\}$ of the coadjoint representation. Let us denote $\mathfrak{U}_{\rho}:\{\mathrm{u} \in$ $\mathfrak{U}: \mathrm{u} \rho=\rho \mathrm{u}\}\left(\rho \in \mathfrak{T}_{s}\right)$. Then $\mathfrak{U}_{\rho}$ is a Lie subgroup of $\mathfrak{U}$, [3, Lemma 2.1.2], and the factorspace $\mathfrak{U} / \mathfrak{U}_{\rho}$ (which can be canonically identified, as a set, with $\mathcal{O}_{\rho}(\mathfrak{U})$ ) endowed with the factor-topology of the analytic Banach Lie group $\mathfrak{U}$ is an analytic Banach manifold, $[6$, III.1.6, Proposition 11]. On the other side, the orbit $\mathcal{O}_{\rho}(\mathfrak{U})$ is naturally a subset of the Banach space $\mathfrak{T}_{s}$ endowed with the norm-topology given by the trace-norm $\|\cdot\|_{1}$. The topology induced on $\mathcal{O}_{\rho}(\mathfrak{U})$ from this B-space topology on $\mathfrak{T}_{s}$ need not coincide with the analytic manifold topology of $\mathfrak{U} / \mathfrak{U}_{\rho}$. It is known that this coincidence is not the case for any $\rho$ with infinite-dimensional range, cf. [3, Proposition 2.1.5]. The coincidence of these two topologies means that the immersed subset $\iota\left(\mathfrak{U} / \mathfrak{U}_{\rho}\right)=\mathcal{O}_{\rho}(\mathfrak{U})$ of $\mathfrak{T}_{s}$ endowed with the topology of $\mathfrak{U} / \mathfrak{U}_{\rho}$ is a submanifold of $\mathfrak{T}_{s}$, or equivalently, that the inclusion mapping $\iota: \mathfrak{U} / \mathfrak{U}_{\rho} \rightarrow \mathfrak{T}_{s}$ (provided that $\iota$ is immersion) is a homeomorphism of $\mathfrak{U} / \mathfrak{U}_{\rho}$ onto the topological subspace $\mathcal{O}_{\rho}(\mathfrak{U}) \subset \mathfrak{T}_{s},[7,5.8 .3]$.

We intend to prove that, for any $\rho=\rho^{*} \in \mathfrak{F}(:=$ the linear space of finite-rank operators in a complex Hilbert space $\mathcal{H})$, the topology induced on the subset $\mathcal{O}_{\rho}(\mathfrak{U}):=\left\{\mathrm{u}_{\rho} \mathrm{u}^{*}\right.$ : $\left.\mathrm{u}^{-1}=\mathrm{u}^{*} \in \mathfrak{U} \subset \mathcal{L}(\mathcal{H})\right\}$ from the overlying (resp. "ambient") Banach space of symmetric trace-class operators $\mathfrak{T}_{s}$ is equivalent to the topology of the set $\mathcal{O}_{\rho}(\mathfrak{U})$ considered as the factor-space $\mathfrak{U} / \mathfrak{U}_{\rho}$. If the inclusion $\iota: \mathfrak{U} / \mathfrak{U}_{\rho} \rightarrow \mathcal{O}_{\rho}(\mathfrak{U}) \subset \mathfrak{T}_{s}, \quad[\mathrm{u}]_{\rho} \mapsto \iota\left([\mathrm{u}]_{\rho}\right):=\mathrm{u} \rho \mathrm{u}^{*}$, where $[\mathrm{u}]_{\rho}:=\left\{\mathrm{v} \in \mathfrak{U}: \mathrm{v} \rho \mathrm{v}^{*}=\mathrm{u} \rho \mathrm{u}^{*}\right\}$ is an (injective) immersion, and if it were also homeomorphism of $\mathfrak{U} / \mathfrak{U}_{\rho}$ onto $\iota\left(\mathfrak{U} / \mathfrak{U}_{\rho}\right)=\mathcal{O}_{\rho}(\mathfrak{U})$, then $\mathcal{O}_{\rho}(\mathfrak{U})$ would be a submanifold of $\mathfrak{T}_{s}$, cf. $[7,5.8 .3]$.

Let us sketch our "strategy" of proving this claim here. It was proved in [3, Proposition 2.1.5], that $\mathcal{O}_{\rho}(\mathfrak{U})$ is an immersed submanifold (i.e. the inclusion $\iota: \mathfrak{U} / \mathfrak{U}_{\rho} \rightarrow \mathcal{O}_{\rho}(\mathfrak{U}) \subset \mathfrak{T}_{s}$ is an immersion, [7, 5.7.1]) of $\mathfrak{T}_{s}$ for $\operatorname{dim}(\rho):=\operatorname{rank}(\rho)<\infty$. We are going to prove that the inverse mapping $\iota^{-1}: \mathcal{O}_{\rho}(\mathfrak{U}) \rightarrow \mathfrak{U} / \mathfrak{U}_{\rho}$ is also continuous. It will be useful to our technique to use the metric-space description of continuity of mappings, i.e. the " $\epsilon \leftrightarrow \delta$ language". It is useful to realize for this that the considered orbits $\mathcal{O}_{\rho}(\mathfrak{U})$ are all (for $\operatorname{dim}(\rho)<\infty)$ Riemann manifolds endowed with strong riemannian metrics, [3, Theorem 2.1.19]. Then the manifold topology is given by the corresponding distance function, $[8$, Proposition 4.64], hence all the considered topologies are metric ones, i.e. the topology on $\mathfrak{U}$ given by the operator norm $\|\mathrm{u}-\mathrm{v}\|$, the riemannian topology on $\mathcal{O}_{\rho}(\mathfrak{U})$ represented by a distance function $d_{\rho}\left(\rho^{\prime}, \mathrm{u} \rho^{\prime} \mathrm{u}^{*}\right),{ }^{3}$ and also the topology of the space $\mathfrak{T}_{s}$ into which is $\mathcal{O}_{\rho}(\mathfrak{U})$ embedded is given by the norm $\left\|\rho^{\prime}-\mathrm{u} \rho^{\prime} \mathrm{u}^{*}\right\|_{1}$.

We have to prove that, for any $\rho^{\prime} \in \mathcal{O}_{\rho}(\mathfrak{U})$, and for an arbitrary (small) $\epsilon^{\prime}>0$ there

\footnotetext{
${ }^{3}$ The distance $d_{\rho}$ will not be explicitly calculated here.
} 
is a $\delta^{\prime}>0$ such that if there is an element $\rho^{\prime \prime} \in \mathcal{O}_{\rho}(\mathfrak{U})$ with $\left\|\rho^{\prime \prime}-\rho^{\prime}\right\|_{1}<\delta^{\prime}$, then it is also $d_{\rho}\left(\rho^{\prime}, \rho^{\prime \prime}\right)<\epsilon^{\prime}$. The projection $\Pi_{\rho}: \mathfrak{U} \rightarrow \mathcal{O}_{\rho}(\mathfrak{U}), \mathrm{u} \mapsto[\mathrm{u}]_{\rho} \approx \mathrm{u} \rho \mathrm{u}^{*}$ is continuous (here $[\mathrm{u}]_{\rho} \approx \mathrm{u} \rho \mathrm{u}^{*}$ means the canonical identification of the left cosets $[\mathrm{u}]_{\rho} \subset \mathfrak{U}$ with their realization as the points u $\rho \mathrm{u}^{*}$ of $\left.\mathcal{O}_{\rho}(\mathfrak{U})\right)$. We can use this continuity to avoid necessity of (possibly complicated) calculation od explicit forms of $d_{\rho}$, cf., however, Proposition 3.2: Since $\Pi_{\rho}$ is uniformly continuous (due to obvious invariance of both metrics), to any $\epsilon^{\prime}>0$ there is an $\epsilon>0$ such that if $\|\mathrm{u}-\mathrm{v}\|<\epsilon$, then also $d_{\rho}\left(\mathrm{u} \rho \mathrm{u}^{*}, \mathrm{v} \rho \mathrm{v}^{*}\right)<\epsilon^{\prime}$. So, if we could find to any $\epsilon>0$ and a $\rho^{\prime} \in \mathcal{O}_{\rho}(\mathfrak{U})$ such a $\delta^{\prime}>0$ that for any $\rho^{\prime \prime}:=\mathrm{u} \rho^{\prime} \mathrm{u}^{*}$ : $\left\|\rho^{\prime}-\rho^{\prime \prime}\right\|_{1}<\delta^{\prime}$ it is possible to find an unitary v such that also $\rho^{\prime \prime}=\mathrm{v} \rho^{\prime} \mathrm{v}^{*}$, and such that also $\left\|I_{\mathcal{H}}-\mathrm{v}\right\|<\epsilon$, then the continuity will be proved. We shall proceed essentially in this way, but to avoid explicit calculation of dependence $\epsilon \mapsto \delta^{\prime}(\epsilon)$, we shall use also another known continuity, namely the continuous dependence of the spectral projections $F_{j}\left(\rho^{\prime \prime}\right)$ of $\rho^{\prime \prime}:=\sum_{j} \lambda_{j} F_{j} \in \mathcal{O}_{\rho}(\mathfrak{U})$ onto the $\rho^{\prime \prime}$ itself. Also the homogeneity of the orbit and of its "ambient" space $\mathfrak{T}_{s}$ will lead to a simplification.

The following lemma provides a reader with a 'freedom' in dealing with various topologies induced on the considered orbits.

2.1 Lemma. The topologies coming from the trace class $B$-space $L^{1}(\mathcal{H})\left(:=\mathfrak{T}(\mathcal{H}) \supset \mathfrak{T}_{s} \supset\right.$ $\left.\mathfrak{F}_{N}\right)$, from the Hilbert-Schmidt B-space $L^{2}(\mathcal{H})\left(:=\mathfrak{H} \supset \mathfrak{H}_{s} \supset \mathfrak{T}_{s} \supset \mathfrak{F}_{N}\right)$, as well as from the $C^{*}$-algebra of all bounded operators $L^{\infty}(\mathcal{H}):=\mathcal{L}(\mathcal{H})\left(\supset \mathcal{L}(\mathcal{H})_{s} \supset \mathfrak{H}_{s} \supset \mathfrak{T}_{s} \supset \mathfrak{F}_{N}\right)$, induced on the subset of symmetric finite rank operators $\mathfrak{F}_{N}$ with a fixed maximal dimension $N$ of their ranges are all equivalent.

Proof. These topologies are equivalent in finite dimensional linear spaces. Explicitly, in our case: Let $N$ be maximal dimension of ranges of the considered operators $A, B \in$ $\mathfrak{F}_{s}, A=A^{*}, B=B^{*}$, hence the ranges of the operators $A-B$ are of maximal dimension $2 N$. The considered topologies are all metric topologies induced on $\mathfrak{F}_{N}$ by the corresponding norms from the "above lying" spaces. The distances between $A$ and $B$ are correspondingly given by $\|A-B\|_{1}:=\operatorname{Tr}|A-B|,\|A-B\|_{2}:=\sqrt{\operatorname{Tr}|A-B|^{2}}$, and $\|A-B\|=:\|A-B\|_{\infty}=$ the maximal eigenvalue of $|A-B|$, where $|A-B|$ denotes the absolute value of the operator $A-B,|A-B|:=\sqrt{(A-B)^{*}(A-B)}$. Generally it is $\|C\|_{\infty} \equiv\|C\| \leq\|C\|_{2} \leq\|C\|_{1}$ for any trace-class operator $C$. Conversely, also due to the mentioned inequalities, one clearly has $\|A-B\|_{2} \leq\|A-B\|_{1} \leq 2 N\|A-B\|_{\infty} \leq$ $2 N\|A-B\|_{2}$ for $A, B \in \mathfrak{F}_{N}$. This shows that all the three metric topologies are on $\mathfrak{F}_{N}$ mutually equivalent.

We shall need a rather indirect, but a quite "faithful" expression for "proximity" of finite-rank operators on the same orbit considered as a subset of the B-space $\mathfrak{T}_{s}$, which 
would be more difficult to express directly with a help the usual norms of their differences. To this end we shall need the following lemma.

2.2 Lemma. Let us consider a subset $\mathfrak{F}_{\sigma}$ of bounded symmetric operators $\rho \in \mathcal{L}(\mathcal{H})$ with a given purely discrete finite spectrum $\sigma:=\left\{\lambda_{0}, \lambda_{1}, \lambda_{2}, \ldots \lambda_{n}\right\} \subset \mathbb{C}$. Their spectral projections $F_{j} \equiv F_{j}(\rho),(j=0,1,2, \ldots n)$ are continuous functions of $\rho \in \mathfrak{F}_{\sigma}$ :

$$
\rho:=\sum_{j=0}^{n} \lambda_{j} \cdot F_{j}
$$

in the operator norm topology of $\mathcal{L}(\mathcal{H})$.

Proof. The spectral projections of any symmetric operator $\rho$ are uniquely determined by that operator, hence for a given spectrum (e.g. $\rho \in \mathfrak{F}_{\sigma}$ ) the projections corresponding to fixed spectral values are uniquely determined functions of the operators $\rho \in \mathfrak{F}_{\sigma}$. By a use of a spectral functional calculus one can choose some functions $p_{j}: \mathbb{R} \rightarrow \mathbb{R}$ such, that $p_{j}\left(\lambda_{k}\right) \equiv \delta_{j k}$. Then $p_{j}(\rho)=F_{j}:=F_{j}(\rho), \forall j$. Let us choose for the functions $p_{j}$ polynomials; we define for any complex $z \in \mathbb{C}$

$$
p_{j}(z):=\prod_{k(\neq j)=0}^{n} \frac{z-\lambda_{k}}{\lambda_{j}-\lambda_{k}}
$$

what gives $p_{j}(\rho)=F_{j}(\rho)$, and the continuity of $\rho \mapsto F_{j}(\rho)$ on (any subset of) $\mathfrak{F}_{\sigma}$ is explicitly seen.

This two Lemmas lead immediately to

2.3 Corollary. The spectral projections $F_{j}$ of finite rank operators $\rho \in \mathfrak{F}_{\sigma} \cap \mathfrak{F}_{N}$ are (on the set $\left.\mathfrak{F}_{\sigma} \cap \mathfrak{F}_{N}\right)$ continuous functions $\rho \mapsto F_{j}(\rho)$ of these operators in any of the considered (i.e. trace, Hilbert-Schmidt, and $\mathcal{L}(\mathcal{H})$ ) topologies (taken independently on the domain-, or range-sides).

We shall use in the following text also the Dirac notation for vectors and operators in a complex Hilbert space: $|x\rangle:=x \in \mathcal{H}$ will denote a vector, $\langle x \mid y\rangle$ is the scalar product of such vectors (linear in the second factor), and $|x\rangle\langle y|$ is the operator of one-dimensional range such, that $|x\rangle\left\langle y\left|: \sum_{j} c_{j}\right| z_{j}\right\rangle \mapsto|x\rangle\left\langle y\left|\cdot \sum_{j} c_{j}\right| z_{j}\right\rangle:=\left(\sum_{j} c_{j}\left\langle y \mid z_{j}\right\rangle\right)|x\rangle$.

The constructions needed in the proof of the main theorem use also a more detailed description of consequences of "proximity" of two projections described in the following

2.4 Lemma. Let $E, F$ be two orthogonal projections of finite-dimensional ranges of equal dimensions $N:=\operatorname{dim} E=\operatorname{dim} F:=\operatorname{Tr}(E)$ in an infinite-dimensional Hilbert space $\mathcal{H}$. 
Assume that $E \wedge F=0$, i.e. the subspaces $\mathcal{E}:=E \mathcal{H}$ and $\mathcal{F}:=F \mathcal{H}$ have no nonzero common vectors. Let us also denote $\mathcal{E} \vee \mathcal{F}:=\mathcal{E}+\mathcal{F}=(E \vee F) \mathcal{H}$ the $2 N$-dimensional linear hull in $\mathcal{H}$ of $\mathcal{E} \cup \mathcal{F}$. Let

$$
\operatorname{Tr}\left[(E-F)^{2}\right] \equiv\|E-F\|_{2}^{2}<2 .
$$

Then:

(i) For any one-dimensional projections given by normalized vectors e $\in \mathcal{E}, f \in \mathcal{F}$ : $|e\rangle\langle e|=: P_{e} \leq E$ (i.e. $\left.P_{e} \cdot E=P_{e}\right)$, and $|f\rangle\langle f|=: P_{f} \leq F$, it is: $P_{e} \cdot F \neq 0$, and $P_{f} \cdot E \neq 0$.

(ii) There exists an orthonormal basis $\left\{e_{j}: j=1,2, \ldots, N:=\operatorname{dim} E\right\} \subset \mathcal{H}$ in $\mathcal{E}$, i.e. $\sum_{j} P_{e_{j}}=E$, such that one can find to it an orthonormal basis of $\mathcal{F}:\left\{f_{j}: j=\right.$ $1,2, \ldots, N\} \subset \mathcal{H}$ (i.e. $\sum_{j} P_{f_{j}}=F$ ), satisfying the relations

$$
P_{f_{j}}\left(E-P_{e_{j}}\right)=0, \quad P_{e_{j}}\left(F-P_{f_{j}}\right)=0, \quad \forall j .
$$

(iii) This means that these orthonormal systems $\left\{e_{j}: j=1,2, \ldots, N\right\}$, and $\left\{f_{j}: j=\right.$ $1,2, \ldots, N\}$, decomposing $E$ and $F$, are in a certain strong sense mutually "affiliated":

$$
F\left|e_{j}\right\rangle=\left|f_{j}\right\rangle\left\langle f_{j} \mid e_{j}\right\rangle, \quad \forall j=1,2, \ldots N, 0 \neq\left\langle f_{j} \mid e_{j}\right\rangle \in \mathbb{C},\left\langle e_{j} \mid e_{j}\right\rangle \equiv 1 \equiv\left\langle f_{j} \mid f_{j}\right\rangle,
$$

i.e. from a specific orthonormal 'decomposition' $\left\{e_{j}: j=1,2, \ldots, N\right\}$ of $\mathcal{E}$ the orthonormal system $\left\{f_{j}: j=1,2, \ldots, N\right\}$ 'decomposing' $\mathcal{F}$ and satisfying (2.3) is obtained, uniquely up to a nonzero numerical factor, simply by element-wise orthogonal projections of $e_{j}$ 's onto $\mathcal{F}:=F \mathcal{H}$.

(iv) The above mentioned specific orthonormal basis $\left\{e_{j}: j=1,2, \ldots, N\right\}$ determines also (up to 'phase factors') an orthonormal basis $\left\{e_{j}^{\perp}: j=1,2, \ldots, N\right\}$ of $\mathcal{E}^{\perp}:=[(E \vee F)-$ $E] \mathcal{H}=\mathcal{E} \vee \mathcal{F} \ominus \mathcal{E}$, such that $f_{j}=\alpha_{j} e_{j}+\beta_{j} e_{j}^{\perp}, \alpha_{j} \cdot \beta_{j} \neq 0,(\forall j)$

Proof.

(i): Let there be a projection $P_{e} \leq E$ such that $P_{e} F=0$. Let $e_{1}:=e$, and let $\left\{e_{j}: j=\right.$ $1,2, \ldots N\}$ be an orthonormal system decomposing $E, E=\sum_{j=1}^{N} P_{e_{j}}$. Then

$$
\operatorname{Tr}(E F)=\operatorname{Tr}\left[\left(E-P_{e}\right) F\right]=\sum_{j=2}^{N} \operatorname{Tr}\left(P_{e_{j}} F\right) \leq N-1,
$$


since always it is $\operatorname{Tr}\left(P_{x} F\right) \leq 1, \forall x \in \mathcal{H}$. The estimate (2.5) would be then in contradiction with the assumption (2.2), since $\operatorname{Tr}\left[(E-F)^{2}\right]=2(N-\operatorname{Tr}(E F))$. Due to the symmetry of the assumed conditions with respect to the exchange $E \leftrightarrow F$, one obtains also $P_{f} E \neq 0$. This implies validity of (i).

(ii): We have to prove existence of the bases $\left\{e_{j}\right\}:=\left\{e_{j}: j=1,2, \ldots N:=\operatorname{dim} E\right\}$, and $\left\{f_{j}: j=1,2, \ldots N=\operatorname{dim} F\right\}$ of $\mathcal{E}$, resp. $\mathcal{F}$ satisfying (2.3).

This means to find an orthonormal basis $\left\{e_{j}: j=1,2, \ldots N\right\}$ of $\mathcal{E}$ such, that its element-wise projections are proportional to $f_{j}$ 's, cf. (2.4). This also means that for such a basis $\left\{e_{j}\right\} \subset \mathcal{E}$ the projections $F\left|e_{j}\right\rangle \in \mathcal{F}$ are nonzero and mutually orthogonal.

The statement (i) ensures that all the projections $F|e\rangle$ of all nonzero vectors $e \in \mathcal{E}$ are nonzero, i.e. that the restriction $E F E \in \mathcal{L}(\mathcal{E})$ of the projector $F$ to the subspace $\mathcal{E} \subset \mathcal{H}$ has trivial kernel: $\operatorname{Ker}_{\mathcal{E}}(E F E)=0$. This implies that the bounded operator $E F E=(F E)^{*} F E$ on $\mathcal{E}$ is strictly positive and there is an orthonormal basis $\left\{e_{j}\right\}$ of $\mathcal{E}$ in which the matrix $\left\langle e_{j}|E F E| e_{k}\right\rangle=\left\langle e_{j}|F| e_{k}\right\rangle$ is diagonal, with strictly positive diagonal elements $\left\|F e_{j}\right\|^{2}$.

Let us define then, e.g., $f_{j}:=\left\|F e_{j}\right\|^{-1} \cdot F e_{j}, j=1,2, \ldots, N$; these elements form the wanted decomposition of $\mathcal{F}$, resp. of the projector $F$ satisfying together with the just found basis $\left\{e_{j}\right\}$ the relations (2.3). This proves (ii).

(iii): That statement is just a rephrasing of (ii); the uniqueness also is seen from (2.3).

(iv): Since each $f_{j} \in \mathcal{F}$ constructed as above is orthogonal to all the $e_{k}(k \neq j)$, and $\left\langle f_{j} \mid e_{j}\right\rangle \neq 0$, but it is also $E^{\perp} f_{j} \neq 0$, with $E^{\perp}:=E \vee F-E, f_{j}$ is expressible in the form

$$
f_{j}:=\alpha_{j} e_{j}+\beta_{j} e_{j}^{\perp}, \forall j
$$

where $e_{j}^{\perp} \in \mathcal{E}^{\perp}:=E^{\perp} \mathcal{H}$ is some normalized vector determined by $f_{j}$ up to a 'phase factor', e.g.: $e_{j}^{\perp}:=\left\|E^{\perp} f_{j}\right\|^{-1} E^{\perp} f_{j}$.

We also see that all $\alpha_{j} \cdot \beta_{j} \neq 0$, since all $f_{j} \notin \mathcal{E}$, but also $f_{j} \notin \mathcal{E}^{\perp}$.

The orthogonality between the vectors $f_{j}$ 's : $\left\langle f_{j} \mid f_{k}\right\rangle \equiv \delta_{j k}$ implies also the orthogonality relations for $e_{j}^{\perp}$ 's: $\left\langle e_{j}^{\perp} \mid e_{k}^{\perp}\right\rangle=\delta_{j k}$.

The following lemma is an illustration of one of the main tools used in the proof of the forthcoming theorem:

2.5 Lemma. Let $E, F$ be two orthogonal projections in an infinite-dimensional complex Hilbert space $\mathcal{H}$ of the same finite dimension $N=\operatorname{Tr}(E)=\operatorname{Tr}(F)$. Let us choose $0<\epsilon<2$, and assume that $N-\operatorname{Tr}(E F)<\epsilon^{2} / 4(<1)$. Then there is a unitary operator $\mathrm{u} \in \mathfrak{U}$ such that $\left\|\mathrm{u}-I_{\mathcal{H}}\right\|<\epsilon$, and that $F=\mathrm{u} E \mathrm{u}^{*}$. 
Proof. Let us denote $Q:=E \wedge F, E^{\prime}:=E-Q, F^{\prime}:=F-Q, N^{\prime}:=\operatorname{Tr}\left(E^{\prime}\right)=\operatorname{Tr}\left(F^{\prime}\right)=$ $N-\operatorname{dim} Q, E^{\prime \perp}:=\left(E^{\prime} \vee F^{\prime}\right)-E^{\prime}, F^{\prime \perp}:=\left(E^{\prime} \vee F^{\prime}\right)-F^{\prime}$. Then $E^{\prime} \wedge F^{\prime}=E^{\prime \perp} \wedge F^{\prime \perp}=0$. We have now also $\operatorname{Tr}\left(E^{\prime \perp}\right)=\operatorname{Tr}\left(F^{\prime \perp}\right)=N^{\prime}=: N^{\prime \perp}$. Moreover,

$$
\begin{array}{r}
1>\epsilon^{2} / 4>N-\operatorname{Tr}(E F)=\frac{1}{2} \operatorname{Tr}\left[(E-F)^{2}\right]=\frac{1}{2} \operatorname{Tr}\left[\left(E^{\prime}-F^{\prime}\right)^{2}\right]= \\
N^{\prime}-\operatorname{Tr}\left(E^{\prime} F^{\prime}\right)=\frac{1}{2} \operatorname{Tr}\left[\left(E^{\perp}-F^{\prime \perp}\right)^{2}\right]=N^{\prime \perp}-\operatorname{Tr}\left(E^{\prime \perp} F^{\perp \perp}\right) .
\end{array}
$$

We can now apply Lemma 2.4 to the both couples, i.e. to $\left(E^{\prime} ; F^{\prime}\right)$, as well as to $\left(E^{\prime \perp} ; F^{\perp}\right)$, of projections. Let $\left\{e_{j}: j=1,2, \ldots N^{\prime}\right\}$, resp. $\left\{e_{j}^{\perp}: j=1,2, \ldots N^{\prime}\right\}$ be the orthonormal decompositions of $\mathcal{E}^{\prime}:=E^{\prime} \mathcal{H}$, resp. of $\mathcal{E}^{\prime \perp}:=\left[E^{\prime} \vee F^{\prime}-E^{\prime}\right] \mathcal{H}$, with the corresponding orthonormal decompositions $\left\{f_{j}: j=1,2, \ldots N^{\prime}\right\}$ of $\mathcal{F}^{\prime}:=F^{\prime} \mathcal{H}$, resp. $\quad\left\{f_{j}^{\perp}: j=1,2, \ldots N^{\prime}\right\}$ of $\mathcal{F}^{\perp}:=F^{\perp \perp} \mathcal{H}$ constructed according to Lemma 2.4. Because the normalized vectors $f_{j}, f_{j}^{\perp}$ remained specified, according to Lemma 2.4, up to arbitrary phase factors, we shall choose them so that the scalar products $\left\langle f_{j} \mid e_{j}\right\rangle>$ $0,\left\langle f_{j}^{\perp} \mid e_{j}^{\perp}\right\rangle>0$. Remember also that for $j \neq k:\left\langle f_{j} \mid e_{k}\right\rangle=0,\left\langle f_{j}^{\perp} \mid e_{k}^{\perp}\right\rangle=0$. We have constructed two orthonormal decompositions of the space $\mathcal{E}^{\prime} \vee \mathcal{F}^{\prime}:=\left(E^{\prime} \vee F^{\prime}\right) \mathcal{H}$, i.e. $\left\{e_{j}, e_{j}^{\perp}: j=1,2, \ldots N^{\prime}\right\}$, as well as $\left\{f_{j}, f_{j}^{\perp}: j=1,2, \ldots N^{\prime}\right\}$. We could also define formally $\left\{e_{j}=f_{j}: j=N^{\prime}+1, \ldots N\right\}$ as an arbitrary orthonormal decomposition of $Q=E-E^{\prime}=F-F^{\prime}$, but it will not be used now.

Let us define now the wanted unitary operator $u \in \mathfrak{U}$ by:

$$
\mathrm{u} x:=x \quad \text { for } x \in \mathcal{H} \ominus\left(\mathcal{E}^{\prime} \vee \mathcal{F}^{\prime}\right) ; \quad \mathrm{u} e_{j}:=f_{j}, \mathrm{u} e_{j}^{\perp}:=f_{j}^{\perp} \quad\left(j=1,2, \ldots N^{\prime}\right),
$$

and this prescription is completed by linearity to a unique unitary operator u on $\mathcal{H}$. Let us prove that this operator has the wanted property. It is clear that $\mathrm{u} E \mathrm{u}^{*}=F: \mathrm{u} E \mathrm{u}^{*}=$ $\mathrm{u} Q \mathrm{u}^{*}+\mathrm{u} E^{\prime} \mathrm{u}^{*}=Q+\sum_{j=1}^{N^{\prime}} \mathrm{u}\left|e_{j}\right\rangle\left\langle e_{j}\left|\mathrm{u}^{*}=Q+\sum_{j=1}^{N^{\prime}}\right| f_{j}\right\rangle\left\langle f_{j}\right|=Q+F^{\prime}=F$. Since on the complement of the finite-dimensional subspace $\mathcal{E}^{\prime} \vee \mathcal{F}^{\prime}$ of $\mathcal{H}$ the operator u coincides with $I_{\mathcal{H}}$, their difference $\mathrm{u}-I_{\mathcal{H}}$ can be nonzero just on the finite dimensional subspace $\mathcal{E}^{\prime} \vee \mathcal{F}^{\prime}$. Hence the norm $\left\|\mathrm{u}-I_{\mathcal{H}}\right\|$ can be calculated as the norm of the restriction to the subspace $\mathcal{E}^{\prime} \vee \mathcal{F}^{\prime}$, and we can deal with this operator $u-I_{\mathcal{H}}$ as with a finite-dimensional matrix. Or, the operator $\mathrm{u}-I_{\mathcal{H}}$ is of finite rank in $\mathcal{H}$. Let us denote $\operatorname{Tr}^{\prime}(C)$ the trace of the restriction of $C \in \mathcal{L}(\mathcal{H})$ to the $2 N^{\prime}$-dimensional subspace $\mathcal{E}^{\prime} \vee \mathcal{F}^{\prime}: \operatorname{Tr}^{\prime}(C):=\operatorname{Tr}\left[\left(E^{\prime} \vee F^{\prime}\right) C\right]$. We have 


$$
\begin{array}{r}
\left\|\mathrm{u}-I_{\mathcal{H}}\right\|^{2} \leq\left\|\mathrm{u}-I_{\mathcal{H}}\right\|_{2}^{2}=\operatorname{Tr}^{\prime}\left[\left(\mathrm{u}^{*}-I_{\mathcal{H}}\right)\left(\mathrm{u}-I_{\mathcal{H}}\right)\right]=\operatorname{Tr}^{\prime}\left[2 I_{\mathcal{H}}-\mathrm{u}-\mathrm{u}^{*}\right]= \\
4 N^{\prime}-\sum_{j=1}^{N^{\prime}}\left[\left\langle e_{j} \mid f_{j}\right\rangle+\left\langle e_{j}^{\perp} \mid f_{j}^{\perp}\right\rangle+\left\langle f_{j} \mid e_{j}\right\rangle+\left\langle f_{j}^{\perp} \mid e_{j}^{\perp}\right\rangle\right]= \\
4 N^{\prime}-2 \sum_{j=1}^{N^{\prime}}\left[\left|\left\langle e_{j} \mid f_{j}\right\rangle\right|+\left|\left\langle e_{j}^{\perp} \mid f_{j}^{\perp}\right\rangle\right|\right],
\end{array}
$$

due to the chosen positivity of the scalar products $\left\langle e_{j} \mid f_{j}\right\rangle,\left\langle e_{j}^{\perp} \mid f_{j}^{\perp}\right\rangle$. According to (2.7), and also from the orthogonality properties of the sets of chosen vectors $\left\{e_{j}, e_{j}^{\perp}, f_{j}, f_{j}^{\perp}\right.$ : $\left.j=1,2, \ldots N^{\prime}\right\}$, and because it is $|\langle e \mid f\rangle|^{2} \leq|\langle e \mid f\rangle| \leq 1$ for scalar product of any two normalized vectors $e, f$ in $\mathcal{H}$, one has

$$
\begin{array}{r}
2 N^{\prime}-\sum_{j=1}^{N^{\prime}}\left[\left|\left\langle e_{j} \mid f_{j}\right\rangle\right|+\left|\left\langle e_{j}^{\perp} \mid f_{j}^{\perp}\right\rangle\right|\right] \leq 2 N^{\prime}-\sum_{j=1}^{N^{\prime}}\left[\left|\left\langle e_{j} \mid f_{j}\right\rangle\right|^{2}+\left|\left\langle e_{j}^{\perp} \mid f_{j}^{\perp}\right\rangle\right|^{2}\right]= \\
\left(N^{\prime}-\operatorname{Tr}\left(E^{\prime} F^{\prime}\right)\right)+\left(N^{\prime \perp}-\operatorname{Tr}\left(E^{\prime \perp} F^{\prime \perp}\right)<\frac{\epsilon^{2}}{2} .\right.
\end{array}
$$

We have obtained, according to (2.9), the wanted estimate $\left\|\mathrm{u}-I_{\mathcal{H}}\right\|^{2}<\epsilon^{2}$.

We are prepared now to prove the regularity of embeddings into $\mathfrak{T}_{s}$ of unitary orbits through finite-rank symmetric operators.

2.6 Theorem. Let $0 \neq \rho=\rho^{*} \in \mathfrak{F}(:=$ the set of all finite-rank operators on $\mathcal{H}), \mathcal{O}_{\rho}(\mathfrak{U}):=$ $\left\{\mathrm{u} \rho \mathrm{u}^{*}: \mathrm{u} \in \mathfrak{U}\right\} \subset \mathfrak{T}_{s}$. The unitary orbit $\mathcal{O}_{\rho}(\mathfrak{U})$ is a regularly embedded [9, p. 550] submanifold of the Banach space $\mathfrak{T}_{s}$ of symmetric trace-class operators endowed with its trace norm.

Proof. The mapping $\Pi_{\rho}: \mathfrak{U} \rightarrow \mathcal{O}_{\rho}(\mathfrak{U}), \mathrm{u} \mapsto \mathrm{u} \rho \mathrm{u}^{*}$ is an analytic submersion [6, III.§1.6, Prop.11], and the inclusion $\iota_{\rho}: \mathcal{O}_{\rho}(\mathfrak{U}) \rightarrow \mathfrak{T}_{s}$ is an injective immersion (cf. [3, Proposition 2.1.5]), hence the composition $\iota_{\rho} \circ \Pi_{\rho}, \mathfrak{U} \rightarrow \mathfrak{T}_{s}$ is continuous. We want to prove, that the inverse (identity) mapping $\iota_{\rho}^{-1}: \mathcal{O}_{\rho}(\mathfrak{U})\left(\subset \mathfrak{T}_{s}\right) \rightarrow \mathcal{O}_{\rho}(\mathfrak{U})\left(:=\mathfrak{U} / \mathfrak{U}_{\rho}\right)$ is also continuous, if the "domain copy" $\mathcal{O}_{\rho}(\mathfrak{U})$ of $\iota_{\rho}^{-1}$ is taken in the relative topology of the corresponding "ambient" space $\mathfrak{T}_{s} \subset L^{1}(\mathcal{H})$. Because of the invariance of all the relevant metrics with respect to the unitary group action (including their invariance in the "ambient" normed spaces), and also because of the continuity of the projection $\Pi_{\rho}$, it suffices to prove the wanted continuity in an arbitrary point $\rho$ of the orbit by showing the following: To any 
positive $\epsilon>0$ one can find a $\delta^{\prime}>0$ such, that if there is some element $\rho^{\prime}=\mathrm{u} \rho \mathrm{u}^{*} \in \mathcal{O}_{\rho}(\mathfrak{U})$ in the $\delta^{\prime}$-neighbourhood of $\rho$ in the space $\mathfrak{T}_{s}:\left\|\rho-\mathrm{u} \rho \mathrm{u}^{*}\right\|_{1}<\delta^{\prime}$, then it is possible to find a unitary $\mathrm{v} \in \mathfrak{U}:\left\|\mathrm{v}-I_{\mathcal{H}}\right\|<\epsilon$, such that $\mathrm{v} \rho \mathrm{v}^{*}=\mathrm{u} \rho \mathrm{u}^{*}$.

Now we can use, for the sake of simplicity of our expression, that the orbit $\mathcal{O}_{\rho}(\mathfrak{U})$ is also a strong riemannian manifold [3, Thm. 2.1.19] with a distance-function $d_{\rho}\left(\rho^{\prime}, \rho^{\prime \prime}\right)$ generating the topology of $\mathfrak{U} / \mathfrak{U}_{\rho}$ (cf. [8, Proposition 4.64]). Now (due to the continuity of $\left.\Pi_{\rho}\right)$, to any $\epsilon^{\prime}>0$ there is an $\epsilon>0$ such that if $\left\|\mathrm{v}-I_{\mathcal{H}}\right\|<\epsilon$, then $d_{\rho}\left(\rho, \mathrm{v} \rho \mathrm{v}^{*}\right)<$ $\epsilon^{\prime}$. We have to prove that, to this $\epsilon$, there exists the corresponding $\delta^{\prime}>0$ such that $\left\|\rho-\mathrm{u} \rho \mathrm{u}^{*}\right\|_{1}<\delta^{\prime} \Rightarrow d_{\rho}\left(\rho, \mathrm{u} \rho \mathrm{u}^{*}\right)<\epsilon^{\prime}$, what means the desired continuity. The proof will be direct: A construction of a unitary $\mathrm{v}:\|\mathrm{v}-I\|<\epsilon$ for any given $\rho^{\prime}=\mathrm{u} \rho \mathrm{u}^{*}$ lying "sufficiently close" to $\rho$ in $\mathfrak{T}_{s}$, such that it is also $\rho^{\prime}=\mathrm{v} \rho \mathrm{v}^{*}$.

Let us write $\rho=\sum_{j=1}^{n} \lambda_{j} E_{j}, 0<n<\infty$, where $\lambda_{j} \neq \lambda_{k}$ for $j \neq k, E_{j}$ are the orthogonal projections of the spectral measure of $\rho=\rho^{*}, 0<\operatorname{dim} E_{j}:=\operatorname{Tr}\left(E_{j}\right)=: N_{j}<$ $\infty(\forall j \neq 0), E_{0}:=I_{\mathcal{H}}-\sum_{j=1}^{n} E_{j}=: I_{\mathcal{H}}-E, \lambda_{0}:=0, \sum_{j=1}^{n} N_{j}=: N$. Let us denote $F_{j}:=\mathrm{u} E_{j} \mathrm{u}^{*}(\forall j)$, hence $\rho^{\prime}:=\mathrm{u} \rho \mathrm{u}^{*}=\sum_{j} \lambda_{j} F_{j}$, and also $F:=\sum_{j=1}^{n} F_{j}$.

It is clear that the nonnegative numbers $N_{j}-\operatorname{Tr}\left(E_{j} F_{j}\left(\rho^{\prime}\right)\right)$ and $N-\operatorname{Tr}\left(E F\left(\rho^{\prime}\right)\right)$ are all continuous functions of $\rho^{\prime}$, and for $\rho^{\prime}=\rho$ they are all zero. This can be seen, e.g. by representing the projection operators $F_{j} \equiv F_{j}\left(\rho^{\prime}\right)$ by polynomials $p_{j}$ of the operators $\rho^{\prime}$, as it was done in Lemma 2.2.

These considerations imply that, for all sufficiently small $\delta^{\prime}>0$, and for all such $\rho^{\prime}=\mathrm{u} \rho \mathrm{u}^{*}$ that $\left\|\rho-\mathrm{u} \rho \mathrm{u}^{*}\right\|_{1}<\delta^{\prime}$, one obtains

$$
\begin{array}{r}
0 \leq N_{j}-\operatorname{Tr}\left(E_{j} F_{j}\left(\rho^{\prime}\right)\right)=: \delta_{j}<1, j=1,2, \ldots n ; \\
0 \leq N-\operatorname{Tr}\left(E F\left(\rho^{\prime}\right)\right)=: \delta<1,
\end{array}
$$

where $\delta, \delta_{j}(j=1,2, \ldots n)$ can be chosen arbitrarily small positive numbers (i.e. they can be bounded from above by arbitrarily small positive upper bounds determining the choice of the mentioned $\delta^{\prime}>0$, what is possible due to the continuous dependence on $\rho^{\prime}$ of the expressions entering into (2.11)).

Let us choose now $0<\epsilon<1$, and assume that the above mentioned $\delta^{\prime}$ is $\mathrm{such}^{4}$ that

$$
\delta \leq \sum_{j=1}^{n} \delta_{j}<\epsilon^{2} / 4
$$

\footnotetext{
${ }^{4}$ We need not here any explicit expression for the dependence $\epsilon \mapsto \delta^{\prime} \equiv \delta^{\prime}(\epsilon)$; it could be 'in principle' obtained, however, from explicit formulas for the functions $\rho^{\prime} \mapsto F_{j}\left(\rho^{\prime}\right)$, e.g. from those given in the proof of Lemma 2.2.
} 
where the first inequality is a consequence of the definitions (2.11).

We shall now construct, for any $\rho^{\prime}=\mathrm{u} \rho \mathrm{u}^{*}$ with $\left\|\rho^{\prime}-\rho\right\|_{1}<\delta^{\prime}$, such a unitary $\mathrm{v} \in \mathfrak{U}$, that $\mathrm{v} \rho \mathrm{v}^{*}=\mathrm{u} \rho \mathrm{u}^{*}$, and simultaneously $\left\|\mathrm{v}-I_{\mathcal{H}}\right\|<\epsilon$.

Let us denote $Q_{j}:=E_{j} \wedge F_{j}, E_{j}^{\prime}:=E_{j}-Q_{j}, F_{j}^{\prime}:=F_{j}-Q_{j}, Q:=E \wedge F, E^{\prime}:=$ $E-Q, F^{\prime}:=F-Q, E^{\prime \perp}:=\left(E^{\prime} \vee F^{\prime}\right)-E^{\prime}=E \vee F-E, F^{\perp}:=\left(E^{\prime} \vee F^{\prime}\right)-F^{\prime}=E \vee F-$ $F, N_{j}^{\prime}:=\operatorname{dim} E_{j}-\operatorname{dim} Q_{j}=\operatorname{dim} E_{j}^{\prime}=\operatorname{dim} F_{j}^{\prime}, N^{\prime}:=\operatorname{dim} E-\operatorname{dim} Q=\operatorname{dim} E^{\prime}=\operatorname{dim} F^{\prime}=$ $\operatorname{dim} E^{\prime \perp}=\operatorname{dim} F^{\prime \perp}$. Observe that $(E-F)^{2}=[(E \vee F-E)-(E \vee F-F)]^{2}=\left(E^{\prime \perp}-F^{\prime \perp}\right)^{2}$. Also it is $\operatorname{Tr}(E F)=\operatorname{Tr}\left(E^{\prime} F^{\prime}+Q\right)=\operatorname{Tr}\left(E^{\prime} F^{\prime}\right)+N-N^{\prime}$, and $\operatorname{dim}(E \vee F)=N+N^{\prime}$. So that we obtain

$$
\operatorname{Tr}\left[(E-F)^{2}\right]=2[N-\operatorname{Tr}(E F)]=\operatorname{Tr}\left[\left(E^{\perp \perp}-F^{\prime \perp}\right)^{2}\right]=2\left[N^{\prime}-\operatorname{Tr}\left(E^{\prime \perp} F^{\prime \perp}\right)\right] .
$$

Now we can apply Lemma 2.4 separately to each of the couples of projections

$$
\left(E_{j}^{\prime} ; F_{j}^{\prime}\right), \quad j=1,2, \ldots n ; \quad\left(E^{\prime \perp} ; F^{\perp \perp}\right)
$$

and construct the orthonormal systems $\left\{e_{k}^{(j)}: k=1,2, \ldots N_{j}^{\prime}\right\}$ forming the convenient bases of every $\mathcal{E}_{j}^{\prime}:=E_{j}^{\prime} \mathcal{H}(j=1,2, \ldots n)$, and also the basis $\left\{e_{k}^{\perp}: k=1,2, \ldots N^{\prime}\right\}$ of $\mathcal{E}^{\perp}:=E^{\perp} \mathcal{H}$, such that their respective orthogonal projections onto the spaces $\mathcal{F}_{j}^{\prime}:=$ $F_{j}^{\prime} \mathcal{H}(j=1,2, \ldots n)$, and $\mathcal{F}^{\perp}:=F^{\perp} \mathcal{H}$, corresponding to the second projection in the considered pair of (2.14), are the orthogonal (and afterwards normalized) bases $\left\{f_{k}^{(j)}\right.$ : $\left.k=1,2, \ldots N_{j}^{\prime}\right\}$ of $\mathcal{F}_{j}^{\prime}(j=1,2, \ldots n)$, and the orthonormal basis $\left\{f_{k}^{\perp}: k=1,2, \ldots N^{\prime}\right\}$ of $\mathcal{F}^{\perp}$. Let us choose any orthonormal bases $\left\{e_{k}^{(j)} \equiv f_{k}^{(j)}: k=N_{j}^{\prime}+1, \ldots N_{j}\right\}$ of all the subspaces $\mathcal{Q}_{j}:=Q_{j} \mathcal{H}, j=1,2, \ldots n$. We have obtained in this way two orthonormal systems $\left\{e_{k}^{(j)}, e_{i}^{\perp}: k=1,2, \ldots N_{j}, j=1,2, \ldots n, i=1,2, \ldots N^{\prime}\right\}$, and $\left\{f_{k}^{(j)}, f_{i}^{\perp}\right.$ : $\left.k=1,2, \ldots N_{j}, j=1,2, \ldots n, i=1,2, \ldots N^{\prime}\right\}$, each forming a basis of the subspace $\mathcal{E} \vee \mathcal{F}:=(E \vee F) \mathcal{H}$. Remember also the "cross-orthogonality" of the mutually "affiliated" orthonormal systems:

$$
\left\langle f_{k}^{(j)} \mid e_{l}^{(j)}\right\rangle=0 \quad(j=1,2, \ldots, n), \quad\left\langle f_{k}^{\perp} \mid e_{l}^{\perp}\right\rangle=0, \quad \text { for } \quad l \neq k \quad(\forall k, l) .
$$

Let also the arbitrary phase factors at the all $f$ 's entering into the orthonormal sets be chosen so that for all possible values of the indices it is

$$
\left\langle f_{l}^{\perp} \mid e_{l}^{\perp}\right\rangle>0, \quad\left\langle f_{k}^{(j)} \mid e_{k}^{(j)}\right\rangle>0
$$


Now we shall define the wanted unitary v: Let the restriction of v to $\mathcal{H} \ominus(\mathcal{E} \vee \mathcal{F}):=$ $(\mathcal{E} \vee \mathcal{F})^{\perp}$ be the identity (i.e. $\left.\left.\vee\right\rceil_{\mathcal{H} \ominus(\mathcal{E} \vee \mathcal{F})}:=I_{\mathcal{H} \ominus(\mathcal{E} \vee \mathcal{F})}\right)$, and its restriction to $\mathcal{E} \vee \mathcal{F}$ is defined as the linear transformation between the constructed orthonormal systems forming two bases in $\mathcal{E} \vee \mathcal{F}$ specified by:

$$
\mathrm{v} e_{k}^{(j)}:=f_{k}^{(j)}, \quad \mathrm{v} e_{i}^{\perp}:=f_{i}^{\perp} ; \quad \forall i, j, k .
$$

It is clear from this definition of $\mathrm{v}$, esp. from (2.17), that $\sum_{j=1}^{n} \lambda_{j} F_{j}=\mathrm{v}\left(\sum_{j=1}^{n} \lambda_{j} E_{j}\right) \mathrm{v}^{*}$, i.e. $\rho^{\prime}=\mathrm{v} \rho \mathrm{v}^{*}$. Let us show next, that $\left\|\mathrm{v}-I_{\mathcal{H}}\right\|<\epsilon$. Since $\left.\left(\mathrm{v}-I_{\mathcal{H}}\right)\right\rceil_{\mathcal{H} \ominus(\mathcal{E} \vee \mathcal{F})}=0$, we shall estimate the Hilbert-Schmidt norm of $\left(\mathrm{v}-I_{\mathcal{H}}\right)$ in the subspace $\mathcal{E} \vee \mathcal{F}$. Let $\operatorname{Tr}^{\prime}(C)$ will be the trace of the restriction of $C \in \mathcal{L}(\mathcal{H})$ to $\mathcal{E} \vee \mathcal{F}$. We obtain with a help of (2.16):

$$
\begin{array}{r}
\left\|\mathrm{v}-I_{\mathcal{H}}\right\|_{2}^{2}=\operatorname{Tr}^{\prime}\left(2 I_{\mathcal{H}}-\mathrm{v}-\mathrm{v}^{*}\right)= \\
2\left(N+N^{\prime}\right)-2 \sum_{j=1}^{n} \sum_{k=1}^{N_{j}}\left\langle f_{k}^{(j)} \mid e_{k}^{(j)}\right\rangle-2 \sum_{j=1}^{N^{\prime}}\left\langle f_{j}^{\perp} \mid e_{j}^{\perp}\right\rangle= \\
2 \sum_{j=1}^{n}\left[N_{j}-\sum_{k=1}^{N_{j}}\left\langle f_{k}^{(j)} \mid e_{k}^{(j)}\right\rangle\right]+2\left[N^{\prime}-\sum_{j=1}^{N^{\prime}}\left\langle f_{j}^{\perp} \mid e_{j}^{\perp}\right\rangle\right] \leq \\
2 \sum_{j=1}^{n}\left[N_{j}-\sum_{k=1}^{N_{j}}\left|\left\langle f_{k}^{(j)} \mid e_{k}^{(j)}\right\rangle\right|^{2}\right]+2\left[N^{\prime}-\sum_{j=1}^{N^{\prime}}\left|\left\langle f_{j}^{\perp} \mid e_{j}^{\perp}\right\rangle\right|^{2}\right]= \\
2 \sum_{j=1}^{n}\left[N_{j}-\operatorname{Tr}\left(E_{j} F_{j}\right)\right]+2\left[N^{\prime}-\operatorname{Tr}\left(E^{\prime \perp} F^{\prime \perp}\right)\right]= \\
2 \sum_{j=1}^{n}\left[N_{j}-\operatorname{Tr}\left(E_{j} F_{j}\right)\right]+2[N-\operatorname{Tr}(E F)],
\end{array}
$$

where we have used again the orthogonality properties (2.15) of the vectors inside each "block" corresponding to $E_{j}, j=1,2, \ldots n$, as well as to $E^{\prime \perp}: \sum_{j=1}^{n} E_{j}+E^{\perp}=E \vee F$, the fact that $|\langle f \mid e\rangle|^{2} \leq|\langle f \mid e\rangle|$ for any normalized vectors $e, f \in \mathcal{H}$, and also the relation (2.13).

Now we shall use the definitions (2.11), and the assumption (2.12). We obtain:

$$
\left\|\mathrm{v}-I_{\mathcal{H}}\right\|^{2} \leq\left\|\mathrm{v}-I_{\mathcal{H}}\right\|_{2}^{2} \leq 2 \sum_{j=1}^{n} \delta_{j}+2 \delta \leq 4 \sum_{j=1}^{n} \delta_{j}<\epsilon^{2},
$$

what is the desired result. 
Hence, each orbit of the coadjoint action of $\mathfrak{U}$ going through density matrices with only finite number of different eigenvalues is a submanifold of $\mathfrak{T}_{s}$ : There is an open neighbourhood of any point $\nu$ of $\mathcal{O}_{\rho}(\mathfrak{U})=\mathfrak{U} / \mathfrak{U}_{\rho}$ which coincides with intersection of the embedded $\mathcal{O}_{\rho}(\mathfrak{U})$ into $\mathfrak{T}_{s}$ with an open neighbourhood of the point $\nu$ in $\mathfrak{T}_{s}$.

Another possibility of proving this theorem is indicated in the next Section, where such a proof for the specific case of $\mathcal{O}_{\rho}(\mathfrak{U}):=P(\mathcal{H})$ is given.

\section{Some other related results}

To give here a proof of the promised closeness of the unitary coadjoint orbit going through any symmetric trace-class operator of finite rank, ${ }^{5}$ we shall use an encoding of the spectral invariants (i.e. the spectra, and their multiplicities) of these operators into finite positive measures on $\mathbb{R}$ :

3.1 Proposition. The unitary orbits $\mathcal{O}_{\rho}(\mathfrak{U})$ for finite-rank $\rho \in \mathfrak{T}_{s}$ are closed subsets of $\mathfrak{T}_{s}$.

Proof. Let us take now the smooth (although differentiability will not be exploited here) numerical functions $\rho \mapsto a_{n}(\rho):=\operatorname{Tr}\left(\rho^{n+2}\right)$ determined for all symmetric trace-class operators $\rho \in \mathfrak{T}_{s}$. It is claimed that fixing the infinite sequence $\left\{a_{n}(\rho), n=0,1,2, \ldots\right\}$ of real numbers one can determine the unitary orbit $\mathcal{O}_{\rho}(\mathfrak{U}) \subset \mathfrak{T}_{s}$ (on which the numbers $a_{n}$ are constant: $\left.a_{n}\left(\mathrm{u} \rho \mathrm{u}^{*}\right) \equiv a_{n}(\rho), \forall \mathrm{u} \in \mathfrak{U}, \rho \in \mathfrak{T}_{s}\right)$ uniquely. This can be seen as follows: The orbit $\mathcal{O}_{\rho}(\mathfrak{U})$ for a finite-rank $\rho$ is determined by the spectral invariants of any $\nu \in \mathcal{O}_{\rho}(\mathfrak{U})$, i.e. by its nonzero eigenvalues and their multiplicities. These might be, however, determined by a measure $\mu_{\rho}$ on $\mathbb{R}$, namely the (not normalized) measure given by the characteristic function $t(\in \mathbb{R}) \mapsto \operatorname{Tr}\left(\rho^{2} e^{i t \rho}\right)$, the moments of which are exactly the numbers $a_{n}(\rho)$. That measure expressed by the nonzero eigenvalues $\lambda_{j}$ of $\rho$, and their multiplicities $m_{j}$, has the form

$$
\mu_{\rho}=\sum_{j} \lambda_{j}^{2} \cdot m_{j} \cdot \delta_{\lambda_{j}},
$$

where $\delta_{\lambda}$ is the Dirac probabilistic measure concentrated in the point $\lambda$. It is clear that this measure $\mu_{\rho}$ determines the orbit uniquely. The uniqueness of the solution of the Hamburger problem of moments (see [10, Theorem X.4, and Example 4 in Chap. X.6]) for the moments given by the sequence $\left\{a_{n}(\rho), n=0,1,2, \ldots\right\}$ proves, that the measure $\mu_{\rho}$ is in turn determined by the sequence $\left\{a_{n}(\rho)\right\}$ uniquely.

\footnotetext{
${ }^{5}$ Compare, however, also Proposition 2.1.5 in [3].
} 
Since the functions $\rho \mapsto a_{n}(\rho)$ are continuous in the trace (and even Hilbert-Schmidt, and on bounded balls in $\mathfrak{T}_{s}$ also in the operator $\mathcal{L}(\mathcal{H})-$ ) topology, the intersection of the (closed) inverse images $a_{n}^{-1}\left[a_{n}(\rho)\right]\left(n \in \mathbb{Z}_{+}\right)$:

$$
\mathcal{O}_{\rho}(\mathfrak{U})=\bigcap_{n=0}^{\infty}\left\{\nu \in \mathfrak{T}_{s}: a_{n}(\nu)=a_{n}(\rho)\right\}
$$

is a closed subset of $\mathfrak{T}_{s}$ in these (induced) topologies.

Next will be given an independent way of proving the above Theorem 2.6, but only for a specific case of the orbit $\mathcal{O}_{\rho}(\mathfrak{U})$ with $\rho=P_{x}$, i.e. for the projective Hilbert space $P(\mathcal{H})$. A use of that method for other orbits $\mathcal{O}_{\rho}(\mathfrak{U})$ would need calculation of the distance functions $d_{\rho}\left(\mathrm{u} \rho \mathrm{u}^{*}, \mathrm{v} \rho \mathrm{v}^{*}\right)$ on the riemannian manifolds $\mathcal{O}_{\rho}(\mathfrak{U})$ for a general $\rho$ of finite range. ${ }^{6}$

3.2 Proposition. The unitary orbit $\mathcal{O}_{\rho}(\mathfrak{U})$ going through a one dimensional projection $\rho:=P_{x}(0 \neq x \in \mathcal{H})$ is a submanifold of (i.e. it is regularly embedded into) the space $\mathfrak{T}_{s}$ of symmetric trace-class operators.

Proof. It is known, that the riemannian distance function on $P(\mathcal{H})$ is (cf., e.g., the formula (3.2.11) in [3]):

$$
d\left(P_{x}, P_{y}\right)=\sqrt{2} \arccos \sqrt{\operatorname{Tr}\left(P_{x} P_{y}\right)} .
$$

On the other hand, the distance between the same projections in the "ambient space" $\mathfrak{T}_{s}$ is

$$
\operatorname{Tr}\left|P_{x}-P_{y}\right|=2\left[1-\operatorname{Tr}\left(P_{x} P_{y}\right)\right]^{1 / 2},
$$

what is easily obtained as the sum of absolute values $\left|\lambda_{1}\right|+\left|\lambda_{2}\right|$ of the two nonzero real eigenvalues (if $P_{x} \neq P_{y}$; choose $\lambda_{1} \geq \lambda_{2}$ ) of $P_{x}-P_{y}$ : Since $\operatorname{Tr}\left(P_{x}-P_{y}\right)=\lambda_{1}+\lambda_{2}=0$, one has $\lambda_{1}=-\lambda_{2}=: \lambda>0$. Because $2 \lambda^{2}=\operatorname{Tr}\left[\left(P_{x}-P_{y}\right)^{2}\right]=2\left[1-\operatorname{Tr}\left(P_{x} P_{y}\right)\right]$, one obtains $\lambda=\sqrt{1-\operatorname{Tr}\left(P_{x} P_{y}\right)}$, hence the result (3.4). We see that these two metrics are mutually equivalent.

This implies that the convergence of some sequence $\left\{P_{y_{n}}: n \in \mathbb{Z}_{+}\right\}$of points of this orbit to a chosen point $P_{x} \in \mathcal{O}_{\rho}(\mathfrak{U})$ in the space $\mathfrak{T}_{s}$ means also its convergence on the orbit $\mathcal{O}_{\rho}(\mathfrak{U})$, what gives the wanted continuity of the inverse $\iota^{-1}$ of the injective immersion (it was proved earlier in [3] that $\iota$ is an immersion) $\iota: \mathfrak{U} / \mathfrak{U}_{P_{x}}=\mathcal{O}_{P_{x}}(\mathfrak{U}) \rightarrow P(\mathcal{H}) \subset \mathfrak{T}_{s}$ (the set $P(\mathcal{H})$ is taken here in the relative topology of $\left.\mathfrak{T}_{s}\right)$. This means, that the injection $\iota$ is a homeomorphism, hence $P(\mathcal{H})$ is a submanifold (cf. [7]) of $\mathfrak{T}_{s}$.

\footnotetext{
${ }^{6}$ Remember that (cf. [3]) for $\rho \in \mathfrak{T}_{s}$ with infinite range the claim of Theorem 2.6 is false!
} 
It might be useful to formulate an easy generalization of Lemma 2.4. One can see that the condition (2.2) of "proximity" of the two projections $E, F$ was used in the proof of that lemma for proving the item (i) only. Assuming the conclusion (i), one can formulate a generalization of Lemma 2.4 valid also for infinite-dimensional projections, and without any restriction to their mutual "proximity":

3.3 Proposition. Let $E, F$ be two orthogonal projections in a separable (real, or complex) Hilbert space $\mathcal{H}$ with mutually isomorphic ranges: $E \mathcal{H} \sim F \mathcal{H}$. Assume that $E \wedge F=0$, and that for any one-dimensional projections $P_{e} \leq E$, and $P_{f} \leq F$ it is

$$
P_{e} \cdot F \neq 0, \quad P_{f} \cdot E \neq 0 .
$$

Let also the spectrum of EFE be pure-point (i.e. the eigenvectors form a basis of $\mathcal{H}$ ).

Then there is an orthonormal decomposition of $E$ to one-dimensional projections $E=\sum_{j} P_{e_{j}}$ (the sum is strongly convergent), to which there is a unique orthogonal one-dimensional decomposition of $F: \sum_{j} P_{f_{j}}=F$ such that

$$
P_{f_{j}} P_{e_{k}}=0 \quad \text { for } j \neq k, \quad P_{f_{j}} P_{e_{j}} \neq 0,
$$

for all values of the indices.

Proof. The validity of the proposition in the case of $\operatorname{dim} E=\operatorname{dim} F<\infty$ is seen from the proof of Lemma 2.4. In our case, the proof of the Lemma 2.4 can be essentially used as a first step for proving our claims also for infinite dimensions. Let $\operatorname{dim} E=\operatorname{dim} F=\infty$. The operator $E F E$ restricted to $\mathcal{E}:=E \mathcal{H}$ has trivial kernel: $\operatorname{Ker}_{\mathcal{E}}(E F E)=0$, due to the assumption (3.5). Let an orthonormal basis in the subspace $\mathcal{E}:=E \mathcal{H}$ consisting of the eigenvectors of $E F E$ be $\left\{e_{j} \in \mathcal{H}: j \in \mathbb{N}\right\}$. It exists because $E F E$ has pure point spectrum. The basis $\left\{e_{j}\right\}$ also determines an orthonormal decomposition $\left\{P_{e_{j}}\right\}$ of $E$. Then the vectors

$$
f_{j}:=\left\|F e_{j}\right\|^{-1} F e_{j}, \quad \forall j \in \mathbb{N}
$$

form an orthonormal system in $\mathcal{F}:=F \mathcal{H}:\left\langle f_{j} \mid f_{k}\right\rangle \propto\left\langle F e_{j} \mid F e_{k}\right\rangle=\left(e_{j}, E F E e_{k}\right)(\forall j, k)$. Let $P_{f_{j}}$ be the one-dimensional orthogonal projections onto subspaces of $\mathcal{H}$ spanned by $f_{j}$ 's, and define $F_{n}:=\sum_{j=1}^{n} P_{f_{j}}(\leq F)$. Let also $E_{n}:=\sum_{j=1}^{n} P_{e_{j}}(\leq E)$.

The projections $E_{n}$ and $F_{n}$ are both (finite) $n$-dimensional and fulfill the assumptions of the proposition (by obvious interchange $E \leftrightarrow E_{n}, F \leftrightarrow F_{n}$ ). Also it is $F e_{j}=F_{n} e_{j}, j=$ $1,2, \ldots, n$, so that the presently defined $P_{f_{j}}$ 's coincide with those obtained according to 
the proof of Lemma 2.4. It is clear that also the orthogonality relations (3.6) are fulfilled. It remains to show that

$$
F=s-\lim _{n \rightarrow \infty} \sum_{j=1}^{n} P_{f_{j}}:=\bigvee_{n=1}^{\infty} F_{n} .
$$

Obviously, it is $\vee_{n=1}^{\infty} F_{n} \leq F$. We have to prove equality in this relation. Assume that there is a one-dimensional projection $P_{f} \leq F$ orthogonal to all $F_{n}: P_{f} \cdot F_{n} \equiv 0$. Since, according to (3.5), $P_{f} E \neq 0$, there is at least one $e_{k}$ contained in the given orthonormal system $\left\{e_{j} \in \mathcal{H}: j \in \mathbb{N}\right\}$ such that $P_{f} e_{k} \neq 0$. But $F P_{f}=P_{f} F=P_{f}$, and any vector $f_{k} \neq 0$ corresponding to $P_{f_{k}}$ is $f_{k} \propto F e_{k}=F_{n} e_{k}(\forall n \geq k)$. Consequently, for all $n \geq k$ it is $P_{f} F_{n} e_{k}=P_{f} F e_{k}=P_{f} e_{k} \neq 0$, what implies $P_{f} \cdot F_{n} \neq 0(n \geq k)$. So that any assumed $P_{f}$ orthogonal to all $F_{n}$ 's does not exist, and the equality in (3.8) holds.

The uniqueness of $\left\{P_{f_{j}} ; j \in \mathbb{N}\right\}$ corresponding to the decomposition $\left\{P_{e_{j}} ; j \in \mathbb{N}\right\}$ of $E$ and determined by eigenvectors $\left|e_{j}\right\rangle$ of $E F E$ in $\mathcal{E}$, with the stated properties follows from the orthogonality relations (3.6): It is obtained by orthogonal projecting of the $e_{j}$ 's onto $\mathcal{F}: f_{j} \propto F e_{j}, \forall j$. This proves the proposition.

To see a rather weak connection of the derived properties of considered projections $E, F$ with their previously discussed mutual "proximity", we shall consider an explicit representation of these projections. It will show also in which way the point-spectrum of the restriction of $E F E$ to $\mathcal{E}:=E \mathcal{H}$ can be made an arbitrary countable subset of the open interval $(0,1) \subset \mathbb{R}$.

3.4 Example. Let $E$ be an orthogonal projection in a complex Hilbert space $\mathcal{H}$ and let $\left\{e_{j}: j \in J\right\}$ (with an index set $J$ of cardinality $\leq \aleph_{0}$ ) be an orthonormal basis in $\mathcal{E}:=E \mathcal{H}$. Let $E^{\perp}$ be another orthogonal projection in $\mathcal{H}$ with the same "dimension $J$ " of $\mathcal{E}^{\perp}:=E^{\perp} \mathcal{H}$ and orthogonal to $E: E \cdot E^{\perp}=0$. Let $\left\{e_{j}^{\perp}: j \in J\right\}$ be an orthonormal basis of $\mathcal{E}^{\perp}$. Let us choose an arbitrary set of complex numbers $\left\{\alpha_{j}, \beta_{j}: j \in J\right\}$ such that $\alpha_{j} \cdot \beta_{j} \neq 0,\left|\alpha_{j}\right|^{2}+\left|\beta_{j}\right|^{2}=1, \forall j$. Let us define in $\mathcal{H}$ vectors $f_{j}:=\alpha_{j} e_{j}+\beta_{j} e_{j}^{\perp}, \forall j \in J$. The vectors $\left\{f_{j}: j \in J\right\}$ form an orthonormal basis in a subspace $\mathcal{F} \subset \mathcal{H}$ with the orthogonal projection $F: F \mathcal{H}=\mathcal{F}$. It is clear that the couple of projections $(E ; F)$ satisfies the assumptions of the Proposition 3.3, and that the specified sets of vectors $\left\{e_{j}: j \in J\right\}$ and $\left\{f_{j}: j \in J\right\}$ determine decompositions of $E$, and $F$, respectively, appearing in the assertions of Proposition 3.3.

Now we see that the spectrum of our positive bounded operator $E F E$ is pure-point and contains the eigenvalues $\left\{\left|\alpha_{j}\right|^{2}: j \in J\right\}$, with the eigenvectors $e_{j}(j \in J)$. But we could choose the $\alpha_{j}$ 's arbitrarily with the only restriction $0<\left|\alpha_{j}\right|<1$. Hence, the 
pure-point spectrum of $E F E$ with $\operatorname{dim}(E)=\aleph_{0}$ can be made, in this way, an arbitrary countable subset of the real interval $(0,1){ }^{7}$

To investigate the question of mutual "proximity" of projectors $E$ and $F$, let us calculate first the distance $\|E-F\|_{2}^{2}=2(N-\operatorname{Tr}(E F))$ in the case of $|J|=N<\infty$. Due to the orthogonality relations (3.6), resp. (2.15), we have (in the Dirac notation) $\operatorname{Tr}(E F)=\sum_{j \in J}\left|\left\langle e_{j} \mid f_{j}\right\rangle\right|^{2}=\sum_{j \in J}\left|\alpha_{j}\right|^{2}$. So that, it is:

$$
0<\|E-F\|_{2}^{2}=2\left(N-\sum_{j \in J}\left|\alpha_{j}\right|^{2}\right)<2 N
$$

where every value of the open interval $(0,2 N)$ can be reached without violating our general specification of $(E ; F)$. General projections of the dimension $N$ could reach all values in the closed interval: $0 \leq\|E-F\|_{2}^{2} \leq 2 N$.

With a help of their chosen representation, we can calculate also the "proximity" of the projections $(E ; F)$ in the operator norm, i.e. $\|E-F\|$, what can be used also if $|J|=\aleph_{0}$. This can be easily done, because the two-dimensional subspaces spanned by the couples of vectors $\left\{e_{j} ; f_{j}\right\}, j \in J$, are all mutually orthogonal. Then the spectrum of $|E-F|$ can be easily calculated: $|E-F|=\sum_{j \in J}\left|P_{e_{j}}-P_{f_{j}}\right|$, the spectrum is (cf. proof of the Proposition 3.2) $\sigma(|E-F|)=\left\{\sqrt{1-\operatorname{Tr}\left(P_{e_{j}} P_{f_{j}}\right)}: j \in J\right\}$, and the norm is

$$
\|E-F\|=\sup _{j \in J}\left\|P_{e_{j}}-P_{f_{j}}\right\|=\sup _{j \in J} \sqrt{1-\operatorname{Tr}\left(P_{e_{j}} P_{f_{j}}\right)}=\sup _{j \in J} \sqrt{1-\left|\alpha_{j}\right|^{2}} .
$$

In this case, it is possible to reach all the values $0<\|E-F\| \leq 1$ for our projections (the equality can be reached for $\operatorname{dim} E=\infty$ only). $\varnothing$

\section{References}

[1] J. E. Marsden, T. S. Ratiu: Introduction to Mechanics and Symmetry, Springer, New York, 1999.

[2] A. A. Kirillov: Elementy teorii predstavleniyi (Elements of Representations Theory), Nauka, Moscow, 1978, Second edition.

\footnotetext{
${ }^{7}$ Let us note that, according to a theorem by Naimark (cf. [11, Chap. 9, Theorem 3.2]), any positive operator $A: 0 \leq A \leq I_{\mathcal{E}}$ defined on a Hilbert space $\mathcal{E}$ can be extended into the form $\left.A=E F E\right\rceil_{\mathcal{E}}$, where $E, F$ are some orthogonal projections in a Hilbert space $\mathcal{H}$, and $\mathcal{E}=E \mathcal{H}$.
} 
[3] P. Bóna: Extended Quantum Mechanics, acta phys. slov. 50 (2000) 1-198; and revised versions in arXiv: math-ph/9909022, resp in http://sophia.dtp.fmph. uniba.sk/ bona/EQM/eqm8a.pdf; the original preprint version is [4].

[4] P. Bóna: Quantum Mechanics with Mean - Field Backgrounds, Preprint No. Ph1091, Comenius University, Faculty of Mathematics and Physics, Bratislava, October 1991.

[5] Anatol Odzijewicz, Tudor S. Ratiu: Banach Lie-Poisson spaces and reduction, arXiv: math.SG/0210207.

[6] N. Bourbaki: Groupes et Algèbres de Lie, Hermann, Paris, 1972; russ. ed. "Mir", Moscow 1976.

[7] N. Bourbaki: Varietes differentielles et analytiques. Fascicule de resultats, Hermann, Paris, 1967 and 1971; Russ. ed. Mir, Moscow, 1975.

[8] J. T. Schwartz: Nonlinear Functional Analysis, Gordon and Breach, New York, 1969.

[9] Y. Choquet-Bruhat, C. DeWitt-Morette, M. Dillard-Bleick: Analysis, Manifolds, and Physics, Revised edition, North-Holland, Amsterdam - New York - Oxford, 1982.

[10] M. Reed, B. Simon: Methods of Modern Mathematical Physics, Vols.I and II, Academic Press, New York - London, 1972 and 1975;

[11] E. B. Davies: Quantum Theory of Open Systems, Academic, New York, 1976.

[12] J. Grabowski, M. Kus̀, G. Marmo, T. Shulman: Geometry of quantum dynamics in infinite dimension, arXiv: math-ph/1711.06486. 